OPEN ACCESS

Edited by:

András Büki,

University of Pécs, Hungary

Reviewed by:

Tamas Doczi

University of Pécs, Hungary

Francesco Sala,

University of Verona, Italy

${ }^{*}$ Correspondence:

Hugues Duffau

h-duffau@chu-montpellier.fr

Specialty section:

This article was submitted to

Applied Neuroimaging,

a section of the journal

Frontiers in Neurology

Received: 12 July 2020

Accepted: 19 October 2020

Published: 12 November 2020

Citation:

Duffau H (2020) Can Non-invasive Brain Stimulation Be Considered to Facilitate Reoperation for Low-Grade

Glioma Relapse by Eliciting

Neuroplasticity?

Front. Neurol. 11:582489.

doi: 10.3389/fneur.2020.582489

\section{Can Non-invasive Brain Stimulation Be Considered to Facilitate Reoperation for Low-Grade Glioma Relapse by Eliciting Neuroplasticity?}

\author{
Hugues Duffau ${ }^{1,2 *}$ \\ ${ }^{1}$ Department of Neurosurgery, Gui de Chauliac Hospital, Montpellier University Medical Center, Montpellier, France, ${ }^{2}$ Team \\ "Plasticity of Central Nervous System, Stem Cells and Glial Tumors," National Institute for Health and Medical Research \\ (INSERM), U1191 Laboratory, Institute of Functional Genomics, University of Montpellier, Montpellier, France
}

Keywords: awake surgery, low-grade glioma, non-invasive brain stimulation, neuroplasticity, transcranial magnetic stimulation

\section{INTRODUCTION}

Diffuse low-grade glioma (LGG, i.e., World Health Organization grade II glioma) is a brain primary neoplasm with a constant invasion along the cerebral connectome and with an inevitable malignant transformation, which results in functional worsening and ultimately in the death of the patient (1). To optimize the oncofunctional balance of therapeutic management, namely, to increase both the overall survival and the quality of life (QoL), the purpose is to achieve an early and maximal safe surgical resection, performed until critical neural networks have been identified by means of intraoperative corticosubcortical direct electrostimulation (DES) mapping in awake patients (2). Indeed, despite the lack of randomized controlled trials, complete LGG removal and, when functionally feasible, supracomplete resection [i.e., with an oncological margin around the FLAIR signal abnormality visible on the pre-operative magnetic resonance imaging (MRI)] led to a significant increase in median survival around 15 years $(3,4)$, while in parallel, electrical-guided surgery allowed a significant reduction of severe persistent deteriorations, even in the so-called "eloquent regions" (5). In fact, mechanisms of neuroplasticity induced by the slow progression of the LGG over the years, explaining why the vast majority of patients do experience only mild (or even no) neurological deficits at diagnosis (usually made because of inaugural seizures), open the door to massive surgical resection in areas deemed to be inoperable in a rigid localizationist view of brain processing, with functional recovery and return to a normal life (6-9). Such a considerable functional redeployment is possible, thanks to an actual meta-networking brain organization, based on dynamic interactions within and between neural circuits subserving sensorimotor, visuospatial, language, cognitive, emotional, and behavioral functions (10).

\section{SURGERY FOR LOW-GRADE GLIOMA AND FUNCTIONAL REARRANGEMENT}

Nonetheless, despite this major improvement of functional and oncological outcomes following LGG surgery in the two past decades (11), because of its intrinsic diffuse nature, LGG cannot be cured, as evidenced by relapse that may arise even many years after supratotal resection (4). As a consequence, reoperation(s) has been advocated in the event of LGG recurrence, with the aim of reducing again the tumor volume and then decreasing the risk of malignant transformation and prolonging overall survival $(12,13)$. Interestingly, in a large series with more than 1,000 patients, it has been demonstrated that repeat surgeries were significantly associated with greater 
survival (3). However, preservation of QoL might seem more uncertain still in case of subsequent surgery, especially when the first resection was interrupted according to individual functional boundaries. Yet, it is worth noting that functional rearrangement has been observed between the first and second intervention, as revealed by intraoperative DES $(14,15)$. Remarkably, such a functional reorganization, likely elicited by the initial operation itself, the post-operative cognitive rehabilitation (16), and the glioma regrowth, enabled an optimization of the extent of resection while avoiding neurological morbidity $(15,17)$.

On the other hand, this reconfiguration over the years is seen only in a subgroup of LGG patients, and reoperation did not permit to perform (supra)marginal resection in all cases because of some limitations of neuroplasticity, especially related to the involvement of the "minimal common brain" (18). This "neural core," with a low interindividual variability (19) and a low plastic potential, is mainly constituted by the input systems (as the visual and somatosensory systems), the output systems (as the pyramidal system), and the subcortical connectivity [as the associative fibers, e.g., the arcuate fasciculus or the inferior fronto-occipital fasciculus (IFOF)]; see a recent probabilistic atlas of brain plasticity (8). Such a limitation of brain adaption in reaction to glioma migration explains why some degrees of cognitive disturbances may be found, despite a normal neurological examination at the standard clinical evaluation, when an objective neuropsychological assessment is performed in LGG patients. These disorders may be identified before any treatment [as semantic impairment if the left IFOF is invaded (20)], or following surgical resection-as subjective empathy changes related to the disconnection of the left cingulum bundle or the right IFOF (21), or lexical access troubles associated to damages of the left inferior longitudinal fasciculus (8). Consequently, neurosurgeons should find the optimal compromise between the dynamics of neural networks allowing compensation after glioma resection and limitations of brain reshaping based on the knowledge of critical cortical hubs and axonal pathways $(22,23)$. To this end, introducing the fourth dimension to optimize the oncofunctional balance over the years in LGG patients led to the proposal of an original paradigm, that is, to consider a multistage surgical approach. This new concept enables to deal with the individual capacity of the central nervous system to reallocate in reaction to slow glioma progression, at least to some extent $(24,25)$.

\section{NON-INVASIVE BRAIN STIMULATION AND NEUROPLASTICITY}

In this setting, the next step would be to try to promote neural redistribution before reoperation in order to increase the likelihood of achieving an improved extent of resection. This facilitation of brain functional rearrangement seems now possible for several reasons. First, mechanisms underlying neuroplasticity after a first glioma surgery start to be better understood, especially thanks to post-operative neuroimaging studies by means of task-based as well as resting functional MRI (fMRI), which showed a balance between recruitment of perilesional areas and involvement of contralesional homologous regions (2628). Second, besides fMRI, which is based on the principle of neurovascular coupling, resulting in serious limitations as a low reliability and the impossibility to distinguish critical areas from those that can be compensated following brain insult (29), transcranial magnetic stimulation (TMS) has been proposed for functional mapping in cerebral tumor patients (30). Indeed, by evoking a magnetic field able to bypass the skull, TMS may excite neurons in a suprathresholded manner and then can elicit neuronal activity: this permits to quantify network properties such as excitability and connectivity or to cause a transitory virtual lesion disrupting ongoing task, as DES, but non-invasively (31). However, in a recent investigation that compared navigated repetitive TMS (rTMS) with intraoperative DES in glioma patients, TMS showed only $81.6 \%$ sensitivity, $59.6 \%$ specificity, $78.5 \%$ positive predictive value, and $64.1 \%$ negative predictive value for pre-operative language mapping (32), confirming that DES remains the criterion standard. Third, beyond pre-surgical planning, TMS has recently been used to study neuroplasticity before and after tumor surgery. In a recent preliminary experience with 18 patients harboring a left glioma, rTMS language mapping has been achieved before a first and then before a second surgery and confirmed a functional reallocation of language sites, with (i) more "language-negative areas" around the neoplasm during the reoperation in patients in whom critical language areas have been found during the first mapping; (ii) more functional reorganization in slow-growing tumors: in other words, these findings support that eloquent regions can leave the tumor area over time, especially in LGG (33). In agreement with $\mathrm{fMRI}$ studies, by generating many language disorders over the right hemisphere, rTMS investigations plead in favor of an active recruitment of the contralesional side to compensate for the glioma growth in the left side (34).

In addition, non-invasive brain stimulation (NBS) techniques by means of rTMS or anodal transcranial direct current stimulation (tDCS), which can actively generate neuromodulation by changing cortical excitability into inhibitory or excitatory direction using magnetic or electric fields, respectively, may enable both to potentiate behavioral performances in healthy volunteers and to facilitate post-lesional neuroplasticity in brain-damaged patients $(35,36)$. Indeed, repeated sessions of NBS over the healthy brain have significantly improved language functions such as speech, semantic fluency, word retrieval, and verbal learning (37-39). Interestingly, this functional improvement was significantly associated with a modulation of the effective connectivity, especially between the left inferior frontal gyrus and the right insula in verb learning facilitation (40). This is in line with DES mapping, which disrupts behavior by stimulating focally an entry door to a larger circuit $(41,42)$; even though the effects of NBS are foremost local, neural activity within the whole network is actually affected. For example, regarding movement, the interhemispheric transcallosal inhibitory effects may be modified by applying tDCS to one primary motor cortex, as it can facilitate the contralateral primary motor cortex through potentiation of interhemispheric interactions (43). The same concept has been utilized in patients with cerebral insult, in particular 
for the therapy of post-stroke aphasia. Indeed, although the actual mechanisms of reorganization elicited by excitatory combined with inhibitory effects of NBS on different nodes of the injured neural networks are still matter of debate, it seems that the complex interactions between the ipsilesional, contralesional, and interhemispheric connectivity may be modulated to facilitate functional compensation (44). For instance, anodal tDCS over the left inferior frontal gyrus resulted in an improvement of speech, naming, and repetition in aphasic patients $(45,46)$. However, because the effect of NBS is not restricted to the stimulated region but also evokes modifications of the functional connectivity in a wider language circuit $(47,48)$, beyond excitatory stimulation to perilesional sites, inhibitory low-frequency rTMS has been performed over contralateral homotopic language regions to facilitate post-stroke recovery $(49,50)$. In the same spirit, NBS has also been used in association with speech therapy to potentiate functional compensation (51).

\section{PERSPECTIVES}

Based on these preliminary results in stroke, it could be considered to use NBS in patients who underwent brain surgery, in addition to functional rehabilitation, which is already known to participate in post-operative network rearrangement $(16,52)$. As mentioned, besides the improvement of QoL, the goal would be to optimize the post-operative functional redeployment and to reopen the door to subsequent surgical resection(s), especially for slow-growing LGG (15). Of note, invasive stimulation has previously been suggested by placing a grid of electrodes over the residual glioma at the end of a first partial resection, in order to perform continuous cortical electrical stimulation simultaneously with behavioral training and then to accelerate plastic reorganization prior to reoperation; however, only five patients have been reported, with a high rate of surgical complications (two infections, one subdural hematoma) due to the invasiveness of this technique (53). Moreover, these findings were not reproduced in the literature. As a consequence, a more reliable and feasible original therapeutic solution in clinical routine might be to develop specific NBS protocols that aim

\section{REFERENCES}

1. Duffau H, editor. Diffuse Low-Grade Gliomas in Adults. 2nd ed. London: Springer (2017). doi: 10.1007/978-3-319-55466-2

2. Duffau H. Diffuse low-grade glioma, oncological outcome and quality of life: a surgical perspective. Curr Opin Oncol. (2018) 30:383-9. doi: 10.1097/CCO.0000000000000483

3. Capelle L, Fontaine D, Mandonnet E, Taillandier L, Golmard JL, Bauchet L, et al. Spontaneous and therapeutic prognostic factors in adult hemispheric World Health Organization Grade II gliomas: a series of 1097 cases: clinical article. J Neurosurg. (2013) 118:1157-68. doi: 10.3171/2013.1.JNS121

4. Duffau H. Long-term outcomes after supratotal resection of diffuse low-grade gliomas: a consecutive series with 11-year follow-up. Acta Neurochir. (2016) 158:51-8. doi: 10.1007/s00701-015-2621-3

5. de Witt Hamer PC, Gil Robles S, Zwinderman A, Duffau H, Berger MS. Impact of intraoperative stimulation brain mapping on glioma surgery outcome: a meta-analysis. J Clin Oncol. (2012) 30:2559-65. doi: 10.1200/JCO.2011.38.4818 pushing away functional nodes to leave the glioma region. Indeed, contrary to the post-stroke aphasic patients, in whom it has been proposed to use inhibitory rTMS over the right hemisphere, particularly the inferior frontal gyrus, in order to facilitate reinforcement within the left damaged language network (51), the main purpose in brain tumor patients would be to favor the recruitment of the contralateral homologous areas, which have been demonstrated by means of fMRI as playing a pivotal role in recovery following a first surgery $(26,54)$. In fact, to increase the extent of resection during a reoperation, NBS could be utilized to inhibit the perilesional critical sites and to force them out of the periphery of the surgical cavity, where the tumor removal was interrupted at the end of the first operation because functional boundaries have been reached. In other words, the ultimate goal would be to change the respective weight of the nodes within a large-scale bilateral functional network, or even to modulate the interactions between brain systems-as it has been evidenced that language compensation after surgery for left LGG might involve non-language functions such as attentional resources, i.e., that picture naming recovery was correlated to the recruitment of the right frontoparietal attentional network (28). This means that such an innovative therapeutic strategy can be conceived only in a dynamic metanetworking account of neural processing, breaking with the traditional dogmatic localizationist theory (10); therefore, a stronger link should be built between cognitive neurosciences (as the new field of connectomics), technical advances in neuromodulation tools (as rTMS and tDCS), and elaboration of original management for glioma patients, based on a better understanding and guidance of interactions between tumor progression and brain adaptation. In this spirit, the next question could be to use NBS with the aim of catalyzing neuroplasticity and optimizing the extent of resection for gliomas involving critical neural networks even before the first surgery.

\section{AUTHOR CONTRIBUTIONS}

The author confirms being the sole contributor of this work and has approved it for publication.
6. Duffau H. Lessons from brain mapping in surgery for low-grade glioma: insights into associations between tumour and brain plasticity. Lancet Neurol. (2005) 4:476-86. doi: 10.1016/S1474-4422(05)70140-X

7. Desmurget M, Bonnetblanc F, Duffau H. Contrasting acute and slowgrowing lesions: a new door to brain plasticity. Brain. (2007) 130:898-914. doi: 10.1093/brain/awl300

8. Herbet G, Maheu M, Costi E, Lafargue G, Duffau H. Mapping neuroplastic potential in brain-damaged patients. Brain. (2016) 139:829-44. doi: 10.1093/brain/awv394

9. Ng S, Herbet G, Moritz-Gasser S, Duffau H. Return to work following surgery for incidental diffuse low-grade glioma: a prospective series with 74 patients. Neurosurgery. (2019) 87:720-9. doi: 10.1093/neuros/nyz513

10. Herbet G, Duffau H. Revisiting the functional anatomy of the human brain: toward a meta-networking theory of cerebral functions. Physiol Rev. (2020) 100:1181-228. doi: 10.1152/physrev.00033.2019

11. Ferracci FX, Duffau H. Improving surgical outcome for gliomas with intraoperative mapping. Expert Rev Neurother. (2018) 18:333-41. doi: 10.1080/14737175.2018.1451329 
12. Martino J, Taillandier L, Moritz-Gasser S, Gatignol P, Duffau H. Reoperation is a safe and effective therapeutic strategy in recurrent WHO grade II gliomas within eloquent areas. Acta Neurochir. (2009) 151:427-36. doi: 10.1007/s00701-009-0232-6

13. Schmidt $\mathrm{MH}$, Berger MS, Lamborn KR, Aldape K, McDermott MW, Prados MD, et al. Repeated operations for infiltrative low-grade gliomas without intervening therapy. J Neurosurg. (2003) 98:1165-9. doi: 10.3171/jns.2003.98.6.1165

14. Southwell DG, Hervey-Jumper SL, Perry DW, Berger MS. Intraoperative mapping during repeat awake craniotomy reveals the functional plasticity of adult cortex. J Neurosurg. (2016) 124:1460-9. doi: 10.3171/2015.5.JNS142833

15. Picart T, Herbet G, Moritz-Gasser S, Duffau H. Iterative surgical resections of diffuse glioma with awake mapping: how to deal with cortical plasticity and connectomal constraints? Neurosurgery. (2018) 85:105-16. doi: 10.1093/neuros/nyy218

16. Duffau H. New philosophy, clinical pearls and methods for intraoperative cognition mapping and monitoring "à la carte" in brain tumor patients. Neurosurgery. in press.

17. Duffau H. The huge plastic potential of adult brain and the role of connectomics: new insights provided by serial mappings in glioma surgery. Cortex. (2014) 58:325-37. doi: 10.1016/j.cortex.2013.08.005

18. Ius T, Angelini E, Thiebaut de Schotten M, Mandonnet E, Duffau H. Evidence for potentials and limitations of brain plasticity using an atlas of functional resectability of WHO grade II gliomas: towards a -minimal common brain. Neuroimage. (2011) 56:992-1000. doi: 10.1016/j.neuroimage.2011.03.022

19. Duffau $\mathrm{H}$. A two-level model of interindividual anatomo-functional variability of the brain and its implications for neurosurgery. Cortex. (2017) 86:303-13. doi: 10.1016/j.cortex.2015.12.009

20. Almairac F, Herbet G, Moritz-Gasser S, de Champfleur NM, Duffau H. The left inferior fronto-occipital fasciculus subserves language semantics: a multilevel lesion study. Brain Struct Funct. (2015) 220:1983-95. doi: 10.1007/s00429-014-0773-1

21. Herbet G, Lafargue G, Moritz-Gasser S, Menjot de Champfleur N, Costi E, Bonnetblanc F, et al. A disconnection account of subjective empathy impairments in diffuse low-grade glioma patients. Neuropsychologia. (2015) 70:165-76. doi: 10.1016/j.neuropsychologia.2015.02.015

22. Sarubbo S, Tate M, De Benedictis A, Merler S, Moritz-Gasser S, Herbet G, et al. Mapping critical cortical hubs and white matter pathways by direct electrical stimulation: an original functional atlas of the human brain. Neuroimage. (2020) 205:116237. doi: 10.1016/j.neuroimage.2019.116237

23. Sarubbo S, Tate M, De Benedictis A, Merler S, Moritz-Gasser S, Herbet G, et al. A normalized dataset of 1821 cortical and subcortical functional responses collected during direct electrical stimulation in patients undergoing awake brain surgery. Data Brief. (2019) 28:104892. doi: 10.1016/j.dib.2019.104892

24. Duffau H, Denvil D, Capelle L. Long term reshaping of language, sensory, and motor maps after glioma resection: a new parameter to integrate in the surgical strategy. J Neurol Neurosurg Psychiatry. (2002) 72:511-6. doi: 10.1136/jnnp.72.4.511

25. Robles SG, Gatignol P, Lehéricy S, Duffau H. Long-term brain plasticity allowing a multistage surgical approach to World Health Organization Grade II gliomas in eloquent areas. J Neurosurg. (2008) 109:615-24. doi: 10.3171/JNS/2008/109/10/0615

26. Vassal M, Charroud C, Deverdun J, Le Bars E, Molino F, Bonnetblanc $\mathrm{F}$, et al. Recovery of functional connectivity of the sensorimotor network after surgery for diffuse low-grade gliomas involving the supplementary motor area. J Neurosurg. (2017) 126:1181-90. doi: 10.3171/2016.4.JNS 152484

27. Deverdun J, van Dokkum LEH, Le Bars E, Herbet G, Mura T, D’Agata $\mathrm{B}$, et al. Language reorganization after resection of low-grade gliomas: an fMRI task based connectivity study. Brain Imaging Behav. (2020) 14:1779-91. doi: 10.1007/s11682-019-00114-7

28. van Dokkum LEH, Moritz Gasser S, Deverdun J, Herbet G, Mura T, D’Agata $\mathrm{B}$, et al. Resting state network plasticity related to picture naming in low-grade glioma patients before and after resection. Neuroimage Clin. (2019) 24:102010. doi: 10.1016/j.nicl.2019.102010

29. Azad TD, Duffau H. Limitations of functional neuroimaging for patient selection and surgical planning in glioma surgery. Neurosurg Rev. (2020) 48:E12. doi: 10.3171/2019.11.FOCUS19769
30. Krieg SM, Shiban E, Buchmann N, Gempt J, Foerschler A, Meyer B, et al. Utility of presurgical navigated transcranial magnetic brain stimulation for the resection of tumors in eloquent motor areas. J Neurosurg. (2012) 116:9941001. doi: 10.3171/2011.12.JNS111524

31. Siebner HR, Hartwigsen G, Kassuba T, Rothwell JC. How does transcranial magnetic stimulation modify neuronal activity in the brain? Implications for studies of cognition. Cortex. (2009) 45:1035-42. doi: 10.1016/j.cortex.2009.02.007

32. Motomura K, Takeuchi H, Nojima I, Aoki K, Chalise L, Iijima K, et al. Navigated repetitive transcranial magnetic stimulation as preoperative assessment in patients with brain tumors. Sci Rep. (2020) 10:9044. doi: 10.1038/s41598-020-65944-8

33. Ille S, Engel L, Albers L, Schroeder A, Kelm A, Meyer B, et al. Functional reorganization of cortical language function in glioma patients-a preliminary study. Front Oncol. (2019) 9:446. doi: 10.3389/fonc.2019.00446

34. Rösler J, Niraula B, Strack V, Zdunczyk A, Schilt S, Savolainen P, et al. Language mapping in healthy volunteers and brain tumor patients with a novel navigated TMS system: evidence of tumor-induced plasticity. Clin Neurophysiol. (2014) 125:526-36. doi: 10.1016/j.clinph.2013.08.015

35. Fiori V, Coccia M, Marinelli CV, Vecchi V, Bonifazi S, Ceravolo MG, et al. Transcranial direct current stimulation improves word retrieval in healthy and nonfluent aphasic subjects. J Cognit Neurosci. (2011) 23:2309-23. doi: 10.1162/jocn.2010.21579

36. Schlaug G, Marchina S, Wan CY. The use of non-invasive brain stimulation techniques to facilitate recovery from post-stroke aphasia. Neuropsychol Rev. (2011) 21:288-301. doi: 10.1007/s11065-011-9181-y

37. Cattaneo Z, Pisoni A, Papagno C. Transcranial direct current stimulation over Broca's region improves phonemic and semantic fluency in healthy individuals. Neuroscience. (2011) 183:64-70. doi: 10.1016/j.neuroscience.2011.03.058

38. Holland R, Leff AP, Josephs O, Galea JM, Desikan M, Price CJ, et al. Speech facilitation by left inferior frontal cortex stimulation. Curr Biol. (2011) 21:1403-7. doi: 10.1016/j.cub.2011.07.021

39. Fiori V, Nitsche M, Iasevoli L, Cucuzza G, Caltagirone C, Marangolo P. Differential effects of bihemispheric and unihemispheric transcranial direct current stimulation in young and elderly adults in verbal learning. Behav Brain Res. (2017) 321:170-5. doi: 10.1016/j.bbr.2016.12.044

40. Fiori V, Kunz L, Kuhnke P, Marangolo P, Hartwigsen G. Transcranial direct current stimulation (tDCS) facilitates verb learning by altering effective connectivity in the healthy brain. NeuroImage. (2018) 181:550-9. doi: 10.1016/j.neuroimage.2018.07.040

41. Mandonnet $\mathrm{H}$, Winkler WA, Duffau H. Direct electrical stimulation as an input gate into brain functional networks: principles, advantages and limitations. Acta Neurochir. (2010) 152:185-93. doi: 10.1007/s00701-009-0469-0

42. Duffau H. Stimulation mapping of white matter tracts to study brain functional connectivity. Nat Rev Neurol. (2015) 11:255-65. doi: 10.1038/nrneurol.2015.51

43. Cabibel V, Muthalib M, Teo WP, Perrey S. High-definition transcranial direct-current stimulation of the right M1 further facilitates left M1 excitability during crossed facilitation. J Neurophysiol. (2018) 119:1266-72. doi: $10.1152 /$ jn.00861.2017

44. Hamilton RH, Chrysikou EG, Coslett B. Mechanisms of aphasia recovery after stroke and the role of noninvasive brain stimulation. Brain Lang. (2011) 118:40-50. doi: 10.1016/j.bandl.2011.02.005

45. Fiori V, Cipollari S, Di Paola M, Razzano C, Caltagirone C, Marangolo P. tDCS stimulation segregates words in the brain: evidence from aphasia. Front Hum Neurosci. (2013) 7:269. doi: 10.3389/fnhum.2013.00269

46. Marangolo P, Fiori V, Calpagnano M, Campana S, Razzano C, Caltagirone C, et al. tDCS over the left inferior frontal cortex improves speech production in aphasia. Front Hum Neurosci. (2013) 7:539. doi: 10.3389/fnhum.2013.00539

47. Meinzer M, Antonenko D, Lindenberg R, Hetzer S, Ulm L, Avirame K, et al. Electrical brain stimulation improves cognitive performance by modulating functional connectivity and task-specific activation. J Neurosci. (2012) 32:18566. doi: 10.1523/JNEUROSCI.4812-11.2012

48. Holland R, Leff AP, Penny WD, Rothwell JC, Crinion J. Modulation of frontal effective connectivity during speech. Neuroimage. (2016) 140:126-33. doi: 10.1016/j.neuroimage.2016.01.037 
49. Barwood CH, Murdoch BE, Whelan BM, Lloyd D, Riek S, O'Sullivan J, et al. The effects of low frequency repetitive transcranial magnetic stimulation (rTMS) and sham condition rTMS on behavioural language in chronic nonfluent aphasia: short term outcomes. NeuroRehabilitation. (2011) 28:113-28. doi: 10.3233/NRE-2011-0640

50. Weiduschat N, Thiel A, Rubi-Fessen I, Hartmann A, Kessler J, Merl P, et al. Effects of repetitive transcranial magnetic stimulation in aphasic stroke: a randomized controlled pilot study. Stroke. (2011) 42:409-15. doi: 10.1161/STROKEAHA.110. 597864

51. Thiel A, Hartmann A, Rubi-Fessen I, Anglade C, Kracht L, Weiduschat $\mathrm{N}$, et al. Effects of noninvasive brain stimulation on language networks and recovery in early poststroke aphasia. Stroke. (2013) 44:2240-6. doi: 10.1161/STROKEAHA.111.0 00574

52. Mosca C, Zoubrinetzy R, Baciub M, Aguilar L, Minotti L, Kahane P, et al. Rehabilitation of verbal memory by means of preserved nonverbal memory abilities after epilepsy surgery. Epilepsy Behav Case Rep. (2014) 2:167-73 doi: $10.1016 /$ j.ebcr.2014.09.002
53. Rivera-Rivera PA, Rios-Lago M, Sanchez-Casarrubios S, Salazar O, Yus M, González-Hidalgo M, et al. Cortical plasticity catalyzed by prehabilitation enables extensive resection of brain tumors in eloquent areas. J Neurosurg. (2017) 126:1323-33. doi: 10.3171/2016.2.JNS152485

54. Coget A, Deverdun J, Bonafé A, van Dokkum L, Duffau H, Molino F, et al. Transient immediate postoperative homotopic functional disconnectivity in low-grade glioma patients. Neuroimage Clin. (2018) 18:656-62. doi: 10.1016/j.nicl.2018.02.023

Conflict of Interest: The author declares that the research was conducted in the absence of any commercial or financial relationships that could be construed as a potential conflict of interest.

Copyright $\odot 2020$ Duffau. This is an open-access article distributed under the terms of the Creative Commons Attribution License (CC BY). The use, distribution or reproduction in other forums is permitted, provided the original author(s) and the copyright owner(s) are credited and that the original publication in this journal is cited, in accordance with accepted academic practice. No use, distribution or reproduction is permitted which does not comply with these terms. 